INRA Prod. Anim.,

2012, 25 (3), 291-304

\title{
Apport d'un modèle du fonctionnement du troupeau pour évaluer la production laitière et l'efficacité alimentaire en élevage caprin
}

\author{
L. PUILLET I,2, M. TICHIT 3,4, O. MARTIN ${ }^{1,2}$, D. SAUVANT 1,2 \\ ${ }^{1}$ INRA, UMR791 Modélisation Systémique Appliquée aux ruminants, \\ 16 rue Claude Bernard, F-75005 Paris, France \\ 2 AgroParisTech, UMR Modélisation Systémique Appliquée aux ruminants, \\ 16 rue Claude Bernard, F-75005 Paris, France \\ ${ }^{3}$ INRA, UMR 1048 Sciences pour l'Action et le Développement Activités Produits Territoires, \\ 16 rue Claude Bernard, F-75005 Paris, France \\ ${ }^{4}$ AgroParisTech, UMR Sciences pour l'Action et le Développement Activités Produits Territoires, \\ 16 rue Claude Bernard, F-75005 Paris, France \\ Courriel :Laurence.Puillet@agroparistech.fr
}

Dans un contexte où la maitrise des charges alimentaires est un enjeu majeur pour la pérennité des élevages caprins, l'efficacité du processus de conversion des aliments en lait par le troupeau est une dimension essentielle de la performance. La simulation informatique du fonctionnement du troupeau caprin constitue une voie pour comprendre les effets des pratiques sur le niveau de production laitière et l'efficacité alimentaire, ainsi que les mécanismes biologiques à l'origine de ces performances.

A l'échelle d'un troupeau, la notion d'efficacité repose sur l'aptitude des animaux à transformer les ressources alimentaires en viande et en lait. L'efficacité du processus de transformation est généralement définie par le ratio entre la quantité de produits et la quantité d'aliments consommés (efficacité alimentaire) ou par le ratio entre la quantité d'aliments consommés et la quantité de produits (indice de consommation). Ces différents critères sont utilisés pour évaluer l'efficacité technique du troupeau. Leur intérêt pour le profit économique en élevage laitier est connu (Vandehaar 1998, par exemple), mais il s'est renouvelé avec les récentes envolées du prix des matières premières. Les élevages caprins intensifs sont particulièrement concernés par cette recherche d'efficacité : leurs charges d'alimentation sont souvent élevées, conséquence d'une consommation importante d'aliments concentrés achetés (Institut de l'Elevage 2008). Un des enjeux importants pour ces systèmes est donc de comprendre comment se construit l'efficacité alimentaire et comment elle peut être modulée par les pratiques de conduite du troupeau.

L'efficacité alimentaire du troupeau laitier se construit à partir des efficacités des femelles qui le composent. L'efficacité d'une femelle laitière est caractérisée par sa variabilité ainsi que par la nécessité de la raisonner à l'échelle de la carrière (Peyraud et al 2009). L'intérêt de la variabilité des carrières animales pour améliorer l'évaluation des systèmes d'élevage a été montré par plusieurs auteurs (Landais 1987, Gibon 1994). Certains proposent même de s'appuyer sur cette variabilité pour cibler certaines pratiques de conduite comme la réforme ou le «flushing» (Lee et al 2009). Les carrières sont le plus souvent évaluées sur la base d'indicateurs comme la production laitière, le nombre de réussites à la reproduction ou l'intervalle entre deux mises bas (Lasseur et Landais 1992, Cournut et Dedieu 2004). Seule l'approche développée par Coulon et al (1993, 1995) a introduit des indicateurs relatifs au fonctionnement biologique des animaux durant leur carrière (poids vif, pathologies) sans les relier aux pratiques. Ainsi, les approches à l'échelle des carrières n'ont jamais considéré les critères liés à l'efficacité alimentaire.

Le développement de la modélisation constitue une opportunité pour intégrer la variabilité individuelle à l'échelle des carrières animales et étudier comment les pratiques modulent les performances du troupeau, notamment l'efficacité alimentaire.

L'objectif de cet article est d'illustrer l'apport d'un simulateur du fonctionnement du troupeau caprin laitier pour mieux comprendre l'effet des pratiques sur l'efficacité du troupeau. La première partie de l'article décrit la structure globale du simulateur, qui intègre la variabilité des réponses animales à l'échelle de leur carrière. Ce simulateur a été développé dans le contexte des élevages caprins de Poitou-Charentes. La deuxième partie de l'article présente les résultats d'un ensemble de simulations testant les effets relatifs et combinés i) du potentiel de production laitière du troupeau, ii) de la gestion de l'alimentation (niveau des apports et découpage du plan d'alimentation) et iii) de la reproduction (durée de la lutte et gestion de l'infertilité) sur la production et l'efficacité du troupeau. Ces résultats concernent principalement la relation entre niveau de production et efficacité alimentaire aux échelles du troupeau et de l'animal ainsi que les mécanismes biologiques à l'origine de ces performances. 


\section{1 / Le simulateur du fonc- tionnement du troupeau caprin laitier}

\section{1 / Architecture générale}

Le modèle conceptuel à la base du simulateur informatique combine deux sous-systèmes, un sous-système biotechnique, à l'origine des réponses animales et un sous-système décisionnel, à l'origine des pratiques de conduite (figure 1).

Le sous-système biotechnique repose sur un ensemble de modèles individuels de chèvre laitière (approche individucentrée). Chaque modèle animal simule les dynamiques biologiques d'une femelle de sa naissance à sa sortie du troupeau en fonction de son potentiel de production laitière et des réponses aux pratiques de reproduction et d'alimentation.

Le sous-système décisionnel repose sur un modèle de gestion technique qui formalise le processus de décision de l'éleveur. Ceci permet d'assurer d'une part, une cohérence entre les différentes opérations techniques sur le troupeau et d'autre part, la généricité du simulateur pour qu'il soit adaptable à différents contextes de production. La première étape du processus de décision est à un niveau stratégique : elle correspond à l'organisation du troupeau en lots fonctionnels pour réaliser un projet de production (Puillet et al 2010). La deuxième étape du processus de décision est à un niveau opérationnel : elle correspond à l'organisation temporelle du fonctionnement des lots fonctionnels sur la base d'une succession de motifs élémentaires de gestion. Un motif élémentaire de gestion comprend le planning des opérations techniques et les règles liées à leur exécution.

La combinaison des modèles animaux et du modèle de gestion technique permet de générer la variabilité individuel-
Figure 1. Modèle conceptuel du fonctionnement du troupeau caprin laitier. Les relations entre le système décisionnel et le système biotechnique, illustrées par les flèches, n'ont été représentées que pour les opérations de renouvellement. Ces relations sont présentes également pour les opérations de reproduction et d'alimentation.

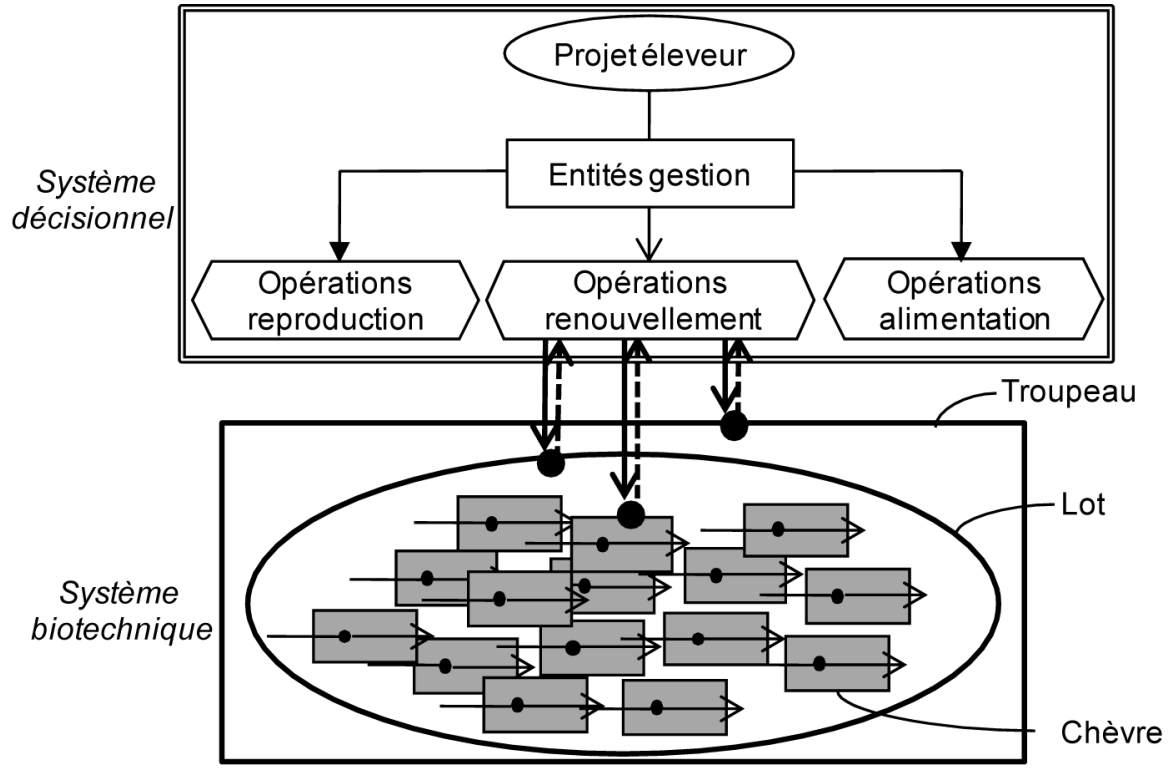

le à l'origine des performances globales $\mathrm{du}$ troupeau. Une première source de variabilité est génétique : le potentiel de production d'un individu est tiré de façon aléatoire dans une distribution statistique qui suit une loi normale (figure 2a). La moyenne de la loi normale correspond au paramètre de potentiel de production moyen du troupeau. L'écart-type de la loi normale représente l'hétérogénéité du troupeau en termes de potentiel de production des animaux: plus l'écart-type est important, plus les individus du troupeau ont des potentiels de production hétérogènes. Une deuxième source de variabilité est liée à l'expression des régulations physiologiques au cours des cycles de gestationlactation. L'initiation d'une gestation est gérée par un tirage aléatoire. Pendant la période de reproduction, pour chaque modèle individuel, l'issue du tirage détermine s'il y a ou non entrée en gestation. Ce processus conduit à une variabilité du démarrage des gestations et donc du déroulement des cycles de gestation-lactation (figure 2b). Cette variabilité des cycles de reproduction génère une variabilité des priorités entre fonctions qui sont à l'origine de la partition de l'énergie ingérée. Ainsi, les réponses à l'énergie ingérée sont également variables (figure $2 \mathrm{c}$ ).

Enfin, une troisième source de variabilité est liée aux actions de conduite, qui sont déclenchées par des indicateurs liés à différents niveaux d'organisation (l'individu, le lot ou le troupeau). Par exemple, l'action de réforme d'un animal se décide au niveau du troupeau (le nombre total de réformes reflète la politique globale de gestion des effectifs) et également au niveau de l'individu (choix d'un individu sur la base de critères individuels comme l'âge). Les pratiques de conduite créent ainsi, pour chaque individu simulé, un microenvironnement au sein du troupeau.

Figure 2. Représentation des sources de variabilité biologique générée par le modèle de fonctionnement du troupeau caprin avec l'exemple de la production laitière : a) potentiel de production, b) date de démarrage du cycle gestation-lactation, c) réponse à l'énergie ingérée.

a)

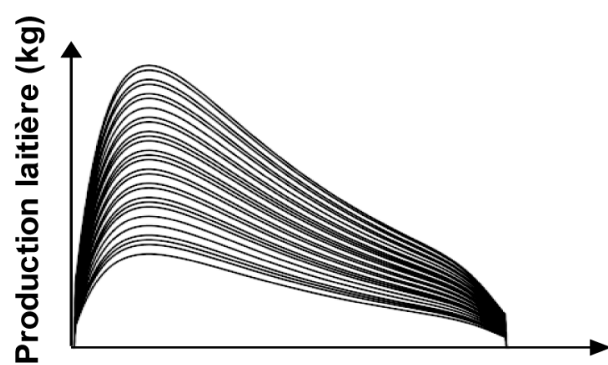

b)

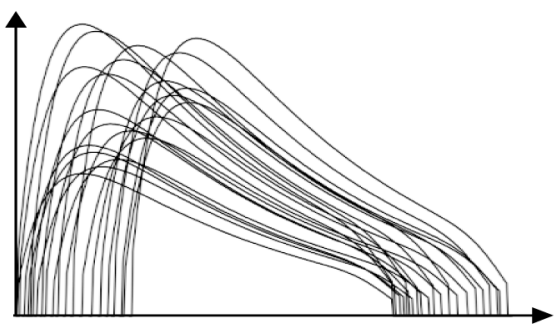

c)

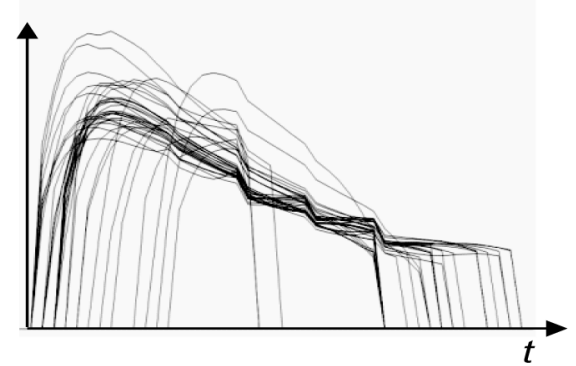


Figure 3. Trajectoire de référence (trait pointillé) et trajectoire réalisée (trait plein) du poids vif simulées par le modèle de chèvre laitière pour une femelle sous-alimentée pendant sa deuxième lactation.

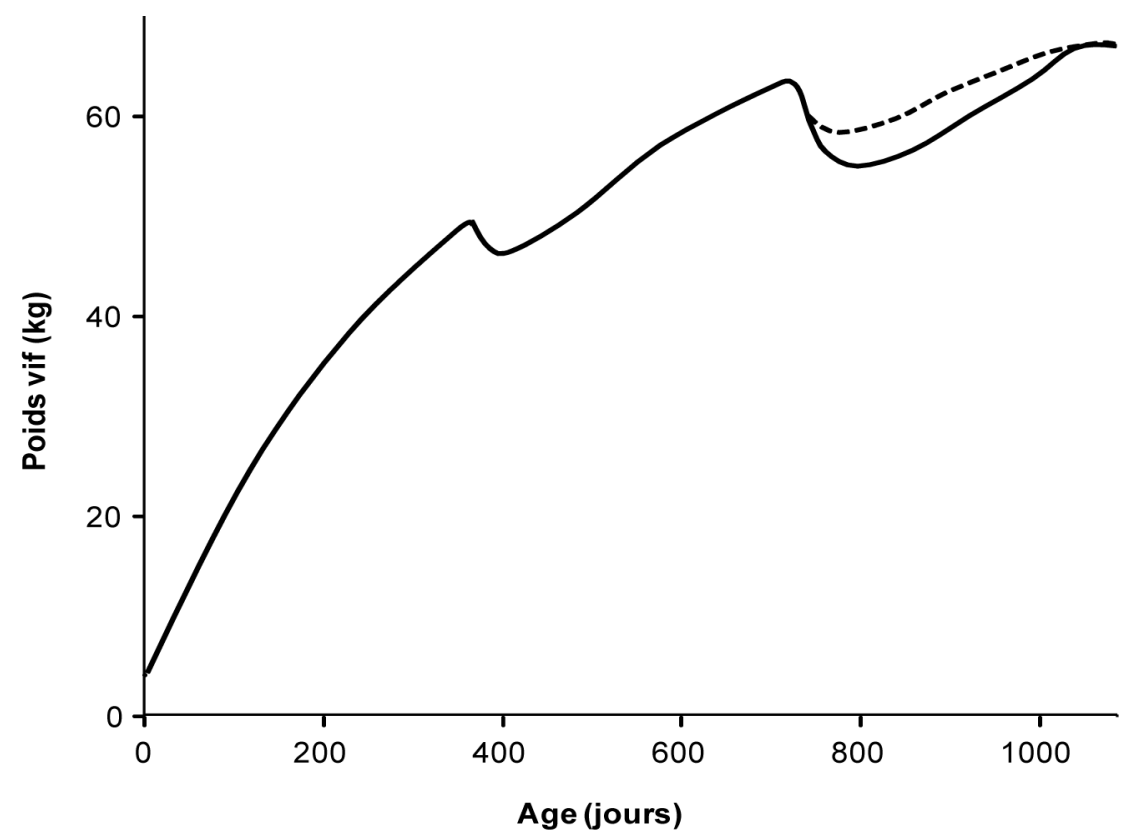

\section{2 / Le modèle de chèvre laitière}

L'entité de base du système biotechnique est un modèle de chèvre laitière qui simule les dynamiques biologiques de la femelle (ingestion, poids vif, production laitière par exemple), en réponse à l'alimentation à l'échelle de sa carrière (Puillet et al 2008, Puillet 2010). Le modèle, cohérent avec les principales recommandations nutritionnelles de l'INRA (Sauvant et al 2007), simule l'allocation d'énergie entre les fonctions physiologiques selon le potentiel de production, les priorités entre fonctions et l'énergie ingérée. Son originalité est d'intégrer une représentation simple des régulations qui déterminent les priorités entre fonctions. Celles-ci pilotent les flux d'énergie associés aux fonctions physiologiques (lactation, utilisation des réserves corporelles...). Les performances simulées reposent sur la notion de trajectoire dite de référence et de trajectoire dite réalisée.

La trajectoire de référence d'une variable individuelle (production laitière, poids vif, par exemple) correspond à l'expression du potentiel de production et des priorités entre fonctions liées à l'enchaînement des cycles de reproduction dans un environnement nutritionnel non contraignant. Pour le poids vif (figure 3), la trajectoire de référence implique une phase de mobilisation, qui est considérée comme pilotée génétiquement et indépendante de l'environnement nutritionnel (Friggens et Newbold 2007). La trajectoire réalisée effectivement résulte des déviations de la trajectoire de référence induites par l'énergie ingérée, fonction de la straté- gie d'alimentation. Lorsque l'énergie ingérée ne permet pas de satisfaire l'expression du potentiel de production, les priorités entre fonctions sont modifiées. Les réserves corporelles ont un rôle important dans la réponse à l'énergie ingérée. Lorsque l'énergie ingérée est en dessous ou au-dessus des besoins, la différence est répartie entre la fonction de lactation et les réserves corporelles. Le niveau des réserves corporelles est cependant borné entre un niveau minimal et maximal par rapport au poids vif (voir Puillet 2010 pour une description complète). Le modèle de chèvre laitière n'est ainsi pas assez robuste pour simuler les réponses à des stratégies de sousalimentation fortes ( $40 \%$ en dessous des besoins) qui dureraient plus d'un an. La calibration et la validation du modèle ont été effectuées sur des données issues des expérimentations de l'UMR MoSAR ainsi que sur des données issues de la littérature (Puillet et al 2008).

\section{3 / Le modèle de gestion tech- nique}

Le modèle de gestion technique repose sur deux éléments conceptuels, le lot fonctionnel et le motif élémentaire de gestion, pour représenter le processus de décision de l'éleveur qui conduit aux opérations techniques sur les animaux. Le lot fonctionnel est une entité de gestion autonome. Il est défini comme un ensemble renouvelé de femelles, pilotées à travers un même corps de règles de gestion de l'alimentation, de la reproduction et du renouvellement, qui définissent les opérations techniques. Le lot fonctionnel peut être constitué par des femelles de renouvellement ou par des femelles en production (i.e. qui ont fait au moins une mise bas). L'organisation du troupeau en lots fonctionnels repose sur un nombre de lots, une fonction assignée à chacun et sur les relations entre les lots. Le plus souvent, en élevage laitier, le projet de production correspond à une courbe de livraison de lait. Ainsi, l'organisation en lots fonctionnels est pilotée par l'organisation des périodes de mise bas. Différentes formes d'organisation du troupeau correspondant à différents projets de production sont présentées sur la figure 4. Du point de vue de la gestion des effectifs, le modèle repose sur les hypothèses suivantes: i) la politique d'effectifs est stable et un nombre constant de femelles sont mises à la lutte chaque année ; ii) le renouvellement est basé sur les femelles nées dans le troupeau (pas d'achat) et iii) le taux de renouvellement détermine le taux de réforme. Le nombre de femelles réformées involontairement (i.e. pour maladie ou mortalité) est fixé. Le nombre de femelles réformées volontairement est ainsi la variable d'ajustement pour maintenir une taille de troupeau constante.

La gestion temporelle des lots fonctionnels implique des éléments de planification et de pilotage des opérations techniques. La planification correspond à un plan prévisionnel qui organise les opérations techniques de façon chronologique et qui les positionne dans le calendrier. Le pilotage correspond aux conditions et aux modalités d'exécution des opérations techniques. La planification et le pilotage des opérations techniques sont représentés grâce au concept de motif élémentaire de gestion. $\mathrm{Ce}$ motif correspond à la séquence minimale d'opérations techniques nécessaires à la réalisation d'un cycle de production, au sein du lot fonctionnel. Ainsi, sur le long terme, le fonctionnement d'un lot fonctionnel est basé sur une succession de motifs élémentaires de gestion. Le tableau 1 récapitule les opérations techniques qui sont intégrées dans le motif élémentaire de gestion.

La représentation des pratiques d'alimentation s'appuie sur les concepts développés pour l'analyse fonctionnelle de l'alimentation, proposés par Guérin et Bellon (1989). En particulier, le modèle intègre explicitement la notion d'animal pilote qui représente la référence utilisée par l'éleveur pour raisonner le niveau des apports distribués à un lot. Dans le modèle, l'animal pilote est défini en termes de potentiel de production, arbitrairement exprimé en kg de lait par jour au pic de la troisième lactation (lactation où le maximum de production est exprimé). Cette 
Figure 4. Représentation de l'organisation du troupeau caprin laitier en Lots Fonctionnels (LF : Lot Fonctionnel de femelles productrices ; LF' : Lot Fonctionnel de femelles de renouvellement) pour trois grands types de projets de production correspondant à trois courbes de livraison de lait du troupeau : a) centrée sur la saison de production au printemps, b) centrée sur la contresaison, à l'automne et c) étalée sur l'année.

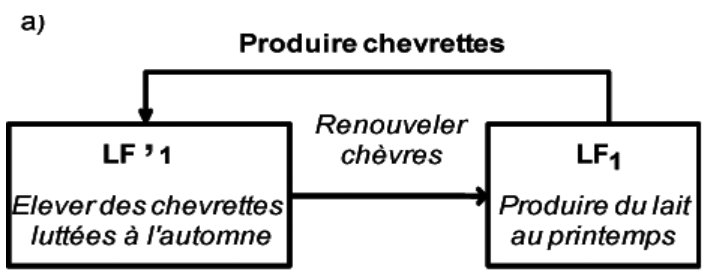

c)

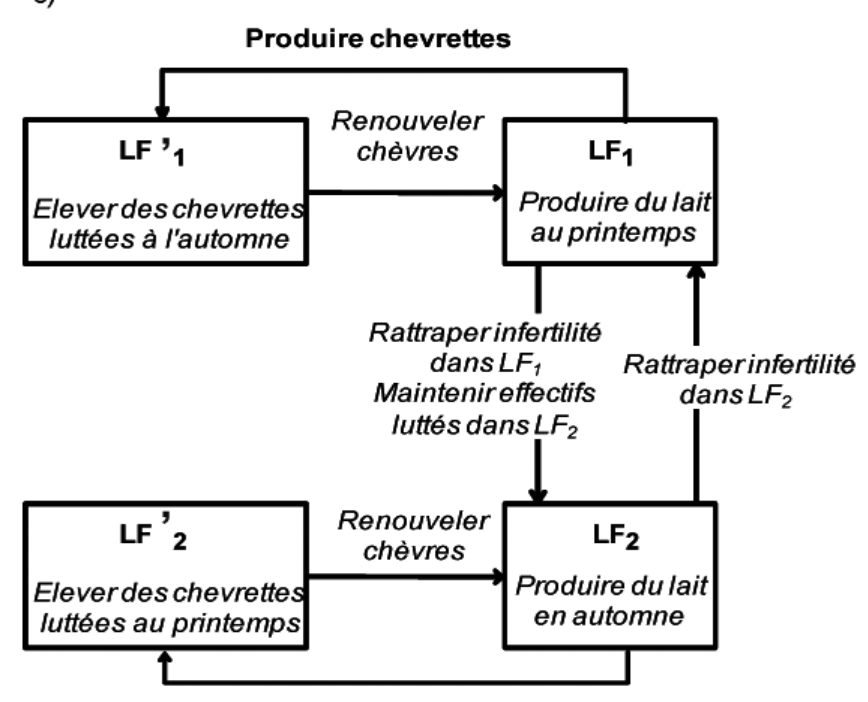

b)

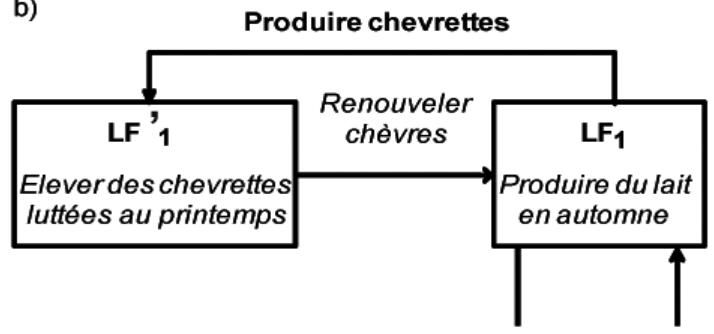

Rattraperles échecs de reproduction de $L F_{1}$

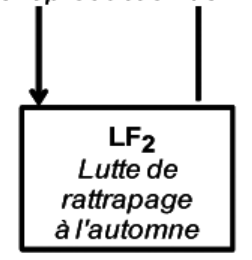

Produire chevrettes

référence détermine la quantité d'aliments concentrés distribuée au lot fonctionnel. Par exemple, un animal pilote fixé à $4 \mathrm{~kg}$ de production potentielle correspond environ à une quantité de $255 \mathrm{~kg}$ d'aliments concentrés distribuée sur 10 mois de lactation. En revanche, un animal pilote fixé à $5 \mathrm{~kg}$ de potentiel de production correspond environ à $310 \mathrm{~kg}$ d'aliments concentrés sur 10 mois de lactation. Le décalage entre l'animal pilote et le niveau de potentiel de production des animaux du lot traduit la gestion du taux de couverture des besoins énergétiques des animaux (figure 5). Ainsi, lorsque le troupeau a un potentiel de production moyen qui est égal à $4 \mathrm{~kg}$ et que l'animal pilote est fixé à $4 \mathrm{~kg}$, les apports alimentaires sont cohérents avec les besoins moyens du lot. La moitié des animaux sont suralimentés et la moitié sont sous-alimentés (figure 5a). En revanche, pour ce même troupeau, dont le potentiel de production moyen est égal à $4 \mathrm{~kg}$, si l'animal pilote est fixé à $5 \mathrm{~kg}$, les apports seront au-dessus des besoins moyens. Une majorité des animaux seront alors suralimentés (figure $5 b$ ). En plus de l'animal pilote pour gérer le niveau des apports alimentaires, la repré-
Figure 5. Représentation de deux stratégies d'alimentation d'un lot d'animaux dont

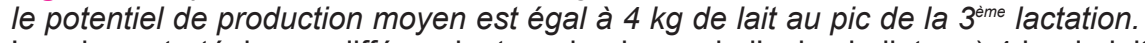
Les deux stratégies se différencient par le niveau de l'animal pilote, a) $4 \mathrm{~kg}$ de lait au pic et b) $5 \mathrm{~kg}$ de lait au pic, qui détermine le niveau des apports alimentaires du lot et la proportion d'animaux sur ou sous-alimentés.

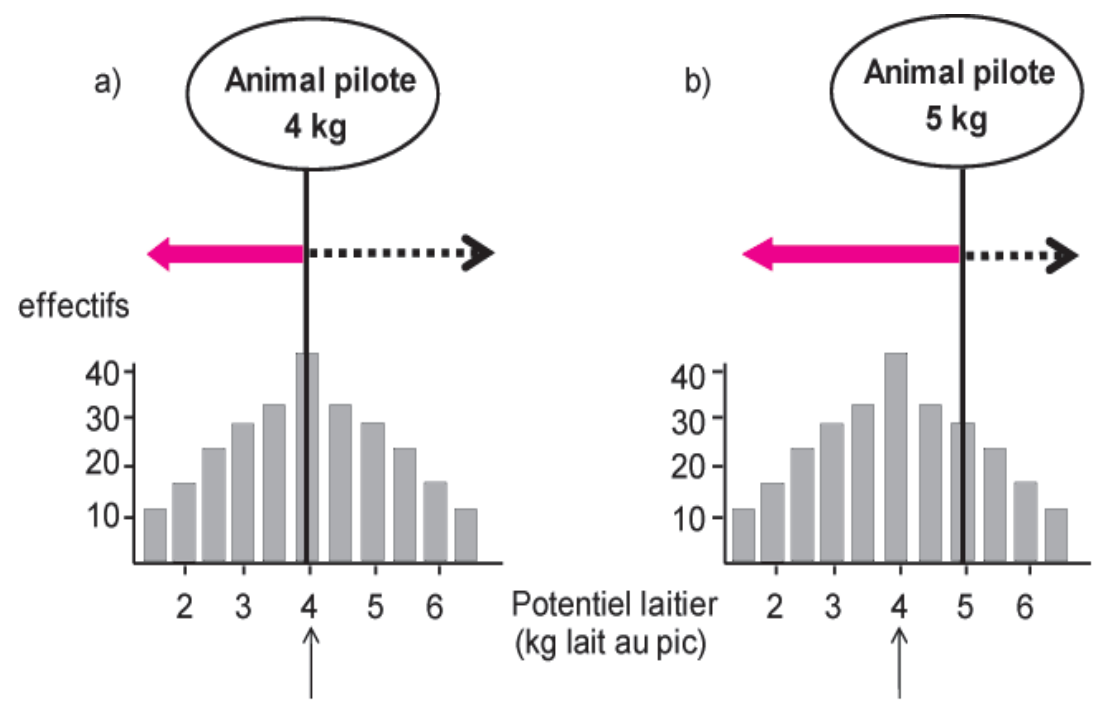

Potentiel de production moyen du lot

Potentiel de production moyen du lot

\section{Animaux suralimentés *...7 Animaux sous-alimentés}


sentation des pratiques d'alimentation intègre une dimension temporelle grâce à la notion de séquence alimentaire. L'enchâ̂nement des séquences alimentaires constitue le plan d'alimentation qui est donc défini par un nombre de séquences et des dates de transition entre les séquences.

Les pratiques de reproduction et de renouvellement sont en partie déterminées à un niveau stratégique, avec l'or- ganisation du troupeau en lots fonctionnels. Le motif élémentaire de gestion précise la durée de la lutte, le taux de réussite du lot qui est le reflet de l'utilisation d'une technique de synchronisation et de saillie, les règles de gestion des chèvres détectées non-gestantes et les critères de réforme volontaire (tableau 1).

Le développement du modèle de gestion technique a reposé principale- ment sur i) un partenariat avec les acteurs de terrain (syndicats de producteurs, recherche, instituts techniques) et ii) le recueil et l'analyse de données produites par différentes structures de recherche-développement (UE INRA Rouillé, Contrôle Laitier, Institut de l'Elevage, BTPL). Ces deux sources d'information ont permis d'identifier les projets de production ainsi que les règles de ges-

Tableau 1. Ensemble des opérations techniques qui constituent le motif élémentaire de gestion dans le simulateur du fonctionnement du troupeau caprin.

\begin{tabular}{|c|c|c|}
\hline Opération technique & Description & Paramétrage \\
\hline Reproduction & $\begin{array}{l}\text { Réalisation du processus de reproduction pendant } \\
\text { la durée de la lutte. Chaque jour de la période } \\
\text { de lutte, un tirage aléatoire détermine pour chaque } \\
\text { chèvre s'il y a entrée en gestation. La probabilité } \\
\text { de succès du tirage dépend de la technique } \\
\text { de synchronisation et du type de saillie. }\end{array}$ & $\begin{array}{l}\text { Durée de la lutte (de } 63 \text { à } 126 \text { jours) } \\
\text { Taux de réussite de la lutte (entre } 70 \\
\text { et } 90 \% \text { de taux de mise bas). }\end{array}$ \\
\hline Diagnostic de gestation & $\begin{array}{l}\text { Calcul de la date de mise bas des chèvres } \\
\text { gestantes et déclenchement des opérations de } \\
\text { gestion des chèvres gestantes et non gestantes. }\end{array}$ & \\
\hline $\begin{array}{l}\text { Gestion des chèvres } \\
\text { gestantes }\end{array}$ & $\begin{array}{l}\text { Calcul de la date de tarissement de chaque chèvre } \\
\text { gestante en fonction de la date prévue de mise } \\
\text { bas ( } 60 \text { jours avant mise bas). }\end{array}$ & \\
\hline $\begin{array}{l}\text { Gestion des chèvres non } \\
\text { gestantes }\end{array}$ & $\begin{array}{l}\text { Application de la modalité de gestion des chèvres } \\
\text { non gestantes définie en entrée du modèle. Les } \\
\text { chevrettes non gestantes sont systématiquement } \\
\text { réformées. }\end{array}$ & \begin{tabular}{|l|} 
Trois modalités de gestion peuvent \\
être définies: \\
- $\quad$ Réforme de toutes les chèvres \\
non gestantes. \\
- \\
Réforme des chèvres non \\
gestantes et avec une production \\
laitière faible; les autres chèvres \\
sont maintenues en lactation \\
longue. \\
- Toutes les chèvres non gestantes \\
sont maintenues en lactation \\
longue.
\end{tabular} \\
\hline Tarissement & Arrêt de la production laitière. & \\
\hline Renouvellement & $\begin{array}{l}\text { Conservation du nombre de chevrettes requis } \\
\text { nées pendant la période de mise bas. Le nombre } \\
\text { de chevrettes gardées est fonction de la taille } \\
\text { du troupeau et du taux de renouvellement } \\
\text { (paramètres d'entrée). }\end{array}$ & Nombre de chevrettes conservées. \\
\hline Intégration du renouvellement & $\begin{array}{l}\text { Changement de lot fonctionnel pour les chevrettes } \\
\text { qui intègrent le lot fonctionnel de femelles } \\
\text { productrices (voir figure } 4 \text { ) juste avant le début } \\
\text { de la période de lutte. }\end{array}$ & \\
\hline Réforme volontaire & $\begin{array}{l}\text { Réforme des chèvres sur la base du niveau de } \\
\text { production et du numéro de lactation. Le nombre } \\
\text { de chèvres réformées volontairement est la } \\
\text { différence entre le nombre de chevrettes de } \\
\text { renouvellement et le nombre de chèvres } \\
\text { réformées involontairement. }\end{array}$ & $\begin{array}{l}\text { Seuil de production laitière. } \\
\text { Seuil de numéro de lactation. }\end{array}$ \\
\hline Passage ration tarissement & $\begin{array}{l}\text { Changement de ration au moment du tarissement. } \\
\text { La composition est définie par l'animal pilote'. }\end{array}$ & Animal pilote (3 à $5 \mathrm{~kg}$ ). \\
\hline $\begin{array}{l}\text { Passage ration lactation } \\
\text { longue }\end{array}$ & $\begin{array}{l}\text { Changement de ration pour les chèvres en } \\
\text { lactation longue. La ration est équivalente à celle } \\
\text { des chèvres qui viennent de mettre bas et définie } \\
\text { par l'animal pilote. }\end{array}$ & Animal pilote (3 à 5 kg). \\
\hline $\begin{array}{l}\text { Passage à la ration } \mathrm{j} \\
\text { (j est compris entre } 1 \\
\text { et le nombre de séquences } \\
\text { alimentaires) }\end{array}$ & $\begin{array}{l}\text { Changement de ration dont la composition est } \\
\text { définie par l'animal pilote. La date du changement } \\
\text { est définie par le nombre de séquences } \\
\text { alimentaires et par la durée de chaque séquence. }\end{array}$ & $\begin{array}{l}\text { Nombre de séquences (1 à } 5) \text {. } \\
\text { Animal pilote ( } 3 \text { à } 5 \mathrm{~kg}) \text {. }\end{array}$ \\
\hline
\end{tabular}

${ }^{1}$ L'animal pilote est exprimé en $\mathrm{kg}$ de lait/jour au pic de la $3^{\text {ème }}$ lactation. II représente l'animal virtuel dont les besoins servent de référence pour déterminer le niveau des apports alimentaires de la ration. 
tion (critères de réforme, de conduite en lactation longue, par exemple) les plus représentatifs des élevages caprins de Poitou-Charentes.

\section{4 / Le simulateur informatique}

Le modèle de chèvre laitière et le modèle de gestion technique ont été implémentés informatiquement avec le logiciel Modelmaker 3.0 (Cherwell Ltd, Oxford, UK, 2000) pour un projet de production de lait au printemps (figure 4a).

Le simulateur informatique est paramétrable pour les principaux critères liés à l'alimentation (nombre de séquences du plan d'alimentation; dates de début et de fin et définition de l'animal pilote pour chaque séquence), à la reproduction (durée de la lutte, taux de réussite et gestion des échecs de reproduction) et au renouvellement (taux et critères de sélection pour la réforme). Les simulations sont conduites sur 20 ans avec un pas de temps journalier. Le simulateur comprend des processus aléatoires (reproduction, par exemple). Il est donc nécessaire de répéter les simulations pour intégrer la variabilité liée à ces processus. Ainsi, chaque scénario simulé est répété 15 fois.

Les 10 dernières années des sorties du simulateur sont analysées. Elles renseignent sur la performance à différents niveaux : $i$ ) évolution temporelle de la structure du troupeau et de ses performances (efficacité et coût alimentaires, production laitière); ii) performances individuelles (production, consommation d'aliments, rythme de reproduction) à l'échelle des carrières, i.e. de la naissance à la sortie du troupeau; iii) indicateurs biologiques individuels (poids vif) à l'échelle de la seconde lactation.

Les sorties du simulateur ont été évaluées par une analyse de sensibilité et discutées avec les partenaires cités plus haut (Puillet et al 2010).

\section{2 / Les effets des pratiques de conduite du troupeau sur la production laitière et l'efficacité alimentaire simulées}

\section{1 / Description des simulations}

Trois plans d'expérience et un total de 14 simulations ont été conçus pour tester les effets de différentes options de conduite sur les performances d'un troupeau de 300 chèvres luttées à l'automne, pendant la saison normale de reproduction (Puillet et al 2011). Les informations relatives au paramétrage des plans d'expérience sont résumées dans le tableau 2. Dans toutes les simu- lations, la reproduction a été paramétrée pour obtenir un bon niveau de réussite (autour de $85 \%$ de taux de mise bas). Ainsi, les effectifs de femelles en lactation, sont comparables entre les simulations ce qui permet d'étudier uniquement les différences liées aux individus. Le taux de renouvellement est identique entre les simulations.

L'objectif du plan d'expérience $n^{\circ} 1$ était de générer différents taux de couverture des besoins des animaux sur la base d'un décalage entre le niveau des apports alimentaires (représenté par l'animal pilote, égal à 4 ou $5 \mathrm{~kg}$ ) et le niveau des besoins (représenté par le potentiel de production moyen du troupeau, égal à 4 ou $5 \mathrm{~kg}$ ).

L'objectif du plan d'expérience $n^{\circ} 2$ était de produire un décalage entre l'évolution dynamique des besoins, générée par l'étalement de la période de reproduction (durée de la lutte égale à 63 ou 126 jours), et l'évolution dynamique des apports, générée par le découpage du plan d'alimentation (2 ou 5 séquences). Dans ce plan d'expérience, l'alimentation est en adéquation avec le potentiel de production du troupeau.

Enfin, l'objectif du plan d'expérience $n^{\circ} 3$ était de tester une conduite simplifiée du troupeau. Nous avons considéré que la simplification portait sur

Tableau 2. Description des plans d'expérience simulés pour un troupeau de 300 chèvres mises à la reproduction à l'automne.

\begin{tabular}{|c|c|c|c|c|c|c|}
\hline Plan d'expérience & $\begin{array}{c}\mathbf{N}^{\circ} \\
\text { simulation }\end{array}$ & $\begin{array}{c}\text { Potentiel } \\
\text { de production } \\
\text { moyen }{ }^{\mathrm{a}} \\
\text { (kg/jour) }\end{array}$ & $\begin{array}{l}\text { Animal } \\
\text { pilote } \\
\text { (kg de } \\
\text { lait/jour) }\end{array}$ & $\begin{array}{c}\text { Nombre } \\
\text { de séquences } \\
\text { du plan } \\
\text { d'alimentation }\end{array}$ & $\begin{array}{l}\text { Durée de la } \\
\text { lutte (jours) }\end{array}$ & $\begin{array}{c}\text { Gestion } \\
\text { des échecs } \\
\text { de reproduction }\end{array}$ \\
\hline \multirow{4}{*}{$\begin{array}{c}1 \\
\text { Décalage entre le niveau } \\
\text { des apports et les besoins } \\
\text { moyens du troupeau }\end{array}$} & S1 & 4 & 4 & \multirow{4}{*}{3} & 84 & Stricte $^{c}$ \\
\hline & S2 & 5 & 4 & & 84 & Stricte \\
\hline & S3 & 4 & 5 & & 84 & Stricte \\
\hline & S4 & 5 & 5 & & 84 & Stricte \\
\hline \multirow{4}{*}{$\begin{array}{l}2 \\
\text { Décalage entre l'étalement } \\
\text { des stades physiologiques } \\
\text { et le découpage du plan } \\
\text { d'alimentation }\end{array}$} & S5 & \multirow{4}{*}{4} & \multirow{4}{*}{4} & 2 & 126 & Stricte \\
\hline & S6 & & & 5 & 126 & Stricte \\
\hline & S7 & & & 2 & 63 & Stricte \\
\hline & S8 & & & 5 & 63 & Stricte \\
\hline \multirow{6}{*}{$\begin{array}{l}3 \\
\text { Conduite simplifiée } \\
\text { (ration unique et maintien } \\
\text { en lactation longue) }\end{array}$} & s9 & \multirow{6}{*}{4} & 4 & \multirow{6}{*}{1} & 126 & Souple $^{d}$ \\
\hline & S10 & & 5 & & 126 & Souple \\
\hline & S11 & & 3 & & 126 & Souple \\
\hline & $\mathrm{S} 12$ & & 4 & & 63 & Souple \\
\hline & $\mathrm{S} 13$ & & 5 & & 63 & Souple \\
\hline & S14 & & 3 & & 63 & Souple \\
\hline
\end{tabular}

${ }^{a}$ Le potentiel de production est défini comme la production laitière au pic de la $3^{\text {ème }}$ lactation (atteinte de la maturité) ; ${ }^{\mathrm{b}} \mathrm{L}$ 'animal pilote est exprimé en $\mathrm{kg}$ de lait/jour au pic de la $3^{\text {eme }}$ lactation. II représente l'animal virtuel dont les besoins servent de référence pour déterminer le niveau des apports alimentaires de la ration ; ${ }^{\mathrm{C}}$ Réforme systématique des femelles ayant échoué après la lutte $;{ }^{d}$ Mise en lactation longue systématique des femelles ayant échoué après la lutte. 
la gestion des échecs de reproduction, les femelles connaissant un échec de reproduction étant maintenues en lactation longue jusqu'à la prochaine opportunité de reproduction. La simplification se traduit également par un plan d'alimentation qui repose sur une ration unique distribuée sur la période de lactation. Trois niveaux d'apports alimentaires et deux niveaux de durée de lutte sont testés dans ce plan pour évaluer quel niveau des apports et quel étalement de la reproduction sont le plus en adéquation avec la conduite simplifiée.

\section{2 / Production et efficacité à l'échelle du troupeau}

La figure 6 présente les résultats des simulations à l'échelle du troupeau pour deux composantes de la performance : le niveau de production laitière et l'efficacité alimentaire.

Ces deux variables correspondent aux moyennes annuelles sur les 10 dernières années de simulation. L'efficacité alimentaire est calculée sur la base des quantités totales annuelles de Matière Sèche (MS) consommées par l'ensemble des animaux du troupeau, chevrettes de renouvellement comprises. Plus la quantité de lait produite par $\mathrm{kg}$ de matière sèche est élevée, plus l'efficacité de transformation des aliments en lait est élevée.

Dans le plan d'expérience $\mathrm{n}^{\circ} 1$, le niveau des apports et le potentiel de production laitier du troupeau ont des effets significatifs sur la production laitière et l'efficacité. Dans les simulations S1 et $\mathrm{S} 2$, les apports alimentaires sont équivalents mais $\mathrm{S} 2$ correspond à un troupeau de potentiel plus élevé : la valorisation des aliments par des animaux de potentiel plus élevé permet d'augmenter conjointement la production et l'efficacité. Cet effet du potentiel de production est confirmé par les résultats de $\mathrm{S} 4$ où le troupeau a un potentiel élevé et où les apports correspondent aux besoins moyens. Les résultats de S4 sont les plus élevés de l'ensemble des simulations. La simulation $\mathrm{S} 3$ où le troupeau a un potentiel moyen avec une alimentation au-dessus des besoins moyens conduit à une efficacité proche de S4 mais avec une production laitière légèrement inférieure. Ainsi, même si le niveau des apports et le potentiel de production du troupeau permettent d'augmenter conjointement la production et l'efficacité du troupeau, la cohérence entre le potentiel moyen et les apports permet des performances plus élevées.

Dans le plan d'expérience $n^{\circ} 2$, le découpage du plan d'alimentation influence significativement la produc- tion laitière et l'efficacité alimentaire. La durée de la lutte et son interaction avec le découpage du plan d'alimentation n'ont d'effet que sur l'efficacité alimentaire. Le découpage fin du plan d'alimentation (5 séquences) augmente conjointement la production et l'efficacité alimentaire. Bien que les effets testés dans le plan $n^{\circ} 2$ soient statistiquement significatifs, leur impact sur les résultats du troupeau reste limité.

Dans le plan d'expérience $n^{\circ} 3$, les résultats montrent des effets significatifs du niveau des apports et de la durée de la lutte. Le niveau des apports a un effet très marqué sur la production et l'efficacité : les simulations sont groupées par rapport au niveau des apports alimentaires (S11 et S14, S9 et S12 et $\mathrm{S} 10$ et S13). Les simulations S9 et S12 correspondent à des troupeaux avec un potentiel laitier moyen et une alimentation au niveau des besoins moyens. Les résultats de la simulation $\mathrm{S} 12$ sont légèrement supérieurs à ceux de $\mathrm{S} 9$. Il semble qu'une durée de lutte plus courte (63 jours pour S12 vs 126 jours pour S9) soit ainsi mieux adaptée à la conduite simplifiée testée dans le plan $n^{\circ} 3$. Le même constat peut être fait pour S11 et S14 : S14 (63 jours de lutte) conduit à une amélioration de l'efficacité et de la production. Cet effet semble même plus marqué que pour S9 par rapport à S12 : la sous-alimentation qui caractérise les simulations S11 et S14 a exacerbé l'effet positif d'un découpage fin de l'alimentation sur la production et l'efficacité. En revanche, pour S10 et S13 la différence n'apparaît pas. Il semble que la suralimentation diminue l'avantage d'un découpage fin du plan d'alimentation.

La tendance globale du nuage de points correspondant aux simulations des 3 plans d'expérience (figure 6) montre que l'efficacité alimentaire du troupeau augmente avec la production laitière du troupeau, avec un gain d'environ $0,03 \mathrm{~kg}$ de lait $/ \mathrm{kg}$ de MS consommé pour un gain de $10000 \mathrm{~kg} / \mathrm{an}$ de lait. Cette tendance traduit un phénomène de dilution des coûts nutritionnels fixes de production (liés à l'entretien des animaux) par la quantité de lait produit. La figure 6 fait apparaître trois groupes distincts de simulations : i) S11 et S14 qui ont généré des productions et des efficacités faibles (production laitière inférieure à $220000 \mathrm{~kg} / \mathrm{an}$; efficacité inférieure à $0,85 \mathrm{~kg}$ de lait $/ \mathrm{kg}$ de MS) ; ii) S1, S2, S5, S6, S7, S8, S9 et $\mathrm{S} 12$ qui ont généré des productions et des efficacités moyennes (production laitière comprise entre 240000 et $270000 \mathrm{~kg} / \mathrm{an}$; efficacité comprise

Figure 6. Production moyenne du troupeau et efficacité alimentaire sur 10 années de simulation pour les 14 simulations présentées dans le tableau 2 ( $\mathbf{\Delta}$ : plan $n^{\circ} 1$; - : plan $n^{\circ} 2$; : : plan $n^{\circ} 3$ ).

Les symboles noirs correspondent aux simulations où le niveau des apports alimentaires correspond aux besoins moyens du troupeau, les symboles gris aux simulations où il y a sous-alimentation et les symboles rouges aux simulations où il y a suralimentation.

L'efficacité alimentaire est calculée sur la base des consommations annuelles d'aliments de tous les animaux (y compris les chevrettes de renouvellement) du troupeau (foin de luzerne et de ray-grass, luzerne déshydratée et concentré énergétique).

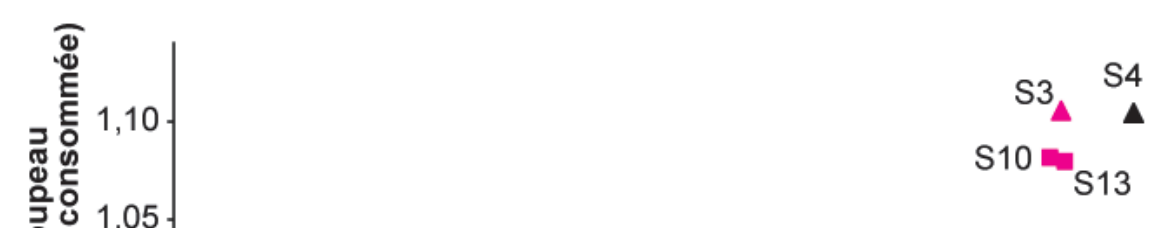

(a)
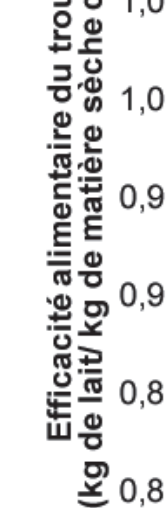

$\stackrel{8}{0} 0,80$

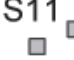
S14

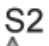

$220000 \quad 240000 \quad 260000 \quad 280000 \quad 300000$

Production laitière du troupeau (kg/an) 
Figure 7. Production moyenne du troupeau et coût alimentaire sur 10 années de simulation pour les 14 simulations présentées dans le tableau 2. ( $\mathbf{\Delta}$ : plan $n^{\circ} 1$; - : plan $n^{\circ} 2$; : : plan $n^{\circ} 3$ ).

Les symboles noirs correspondent aux simulations où le niveau des apports alimentaires correspond aux besoins moyens du troupeau, les symboles gris aux simulations où il y a sous-alimentation et les symboles rouges aux simulations où il y a suralimentation.

Le coût alimentaire est calculé sur la base des consommations annuelles d'aliments de tous les animaux (y compris les chevrettes de renouvellement) du troupeau (foin de luzerne et de ray-grass, luzerne déshydratée et concentré énergétique) et des prix de ces aliments en $2008(0,06 € / \mathrm{kg}$ de matière sèche de fourrage ; $0,23 € / \mathrm{kg}$ de matière sèche d'aliment déshydraté et $0,30 € / \mathrm{kg}$ de matière sèche d'aliment concentré ; source : Institut de l'Elevage, 2008).

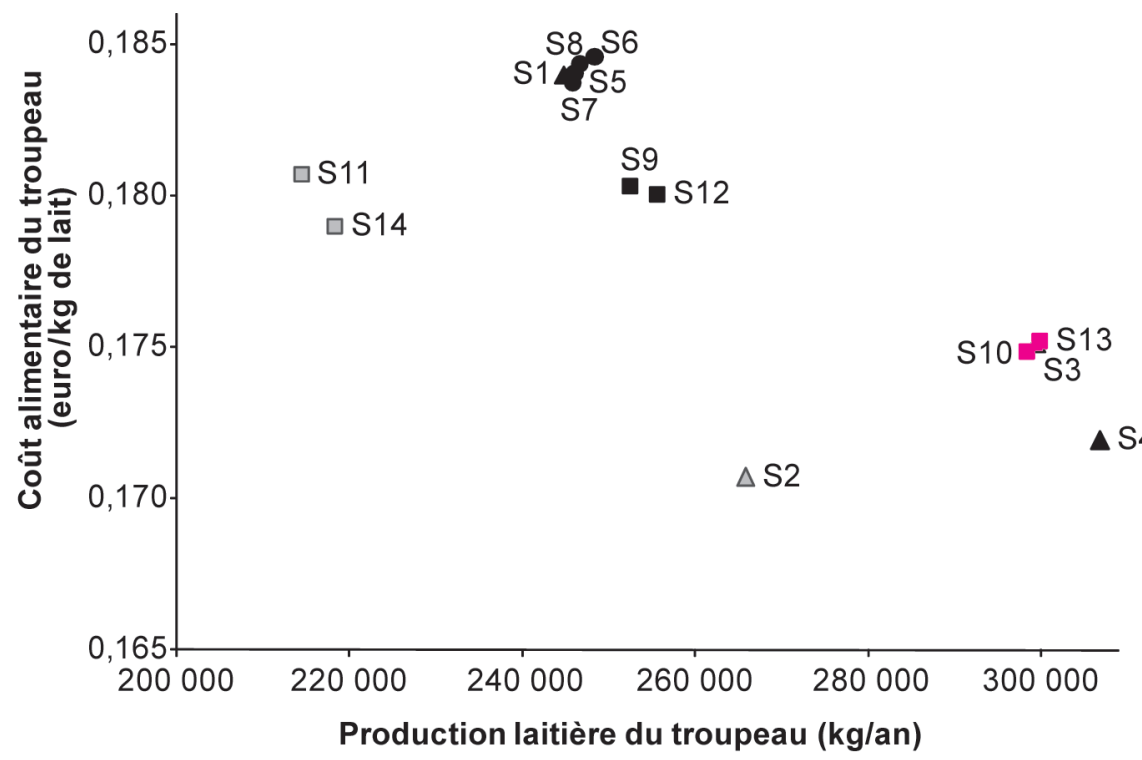

entre 0,90 et $1,00 \mathrm{~kg}$ de lait $/ \mathrm{kg}$ de MS) et iii) S3, S4, S10 et S13 qui ont généré des productions et des efficacités élevées (production laitière supérieure à $290000 \mathrm{~kg} / \mathrm{an}$; efficacité supérieure à $1,05 \mathrm{~kg}$ de lait $/ \mathrm{kg}$ de MS). Les résultats contrastés de ces trois groupes de simulations sont à relier au niveau des apports alimentaires (S11 et S14 : animal pilote égal à $3 \mathrm{~kg} ; \mathrm{S} 1, \mathrm{~S} 2, \mathrm{~S} 5, \mathrm{~S} 6$, $\mathrm{S} 7, \mathrm{~S} 8, \mathrm{~S} 9$ et $\mathrm{S} 12$ : animal pilote égal à $4 \mathrm{~kg}$ et S3, S4, S10 et S13 : animal pilote égal à $5 \mathrm{~kg}$ ). Ensuite, au sein d'un même groupe et donc à même niveau d'apports, le facteur discriminant est le potentiel de production laitier moyen du troupeau (par exemple S2 vs S1, S5, S6, S7, S8, S9 et S12).

La notion d'efficacité alimentaire utilisée pour analyser les résultats du simulateur ne prend pas en compte la composition de la matière sèche consommée. Le simulateur génère pourtant en sortie les consommations d'aliments concentrés, déshydratés et de fourrages. Pour prendre en compte le type d'aliment qui compose l'efficacité, le coût alimentaire du $\mathrm{kg}$ de lait produit a été calculé sur la base des prix des aliments en 2008 . L'objectif ici est d'illustrer comment le coût alimentaire permet de compléter l'évaluation des effets des pratiques sur la production et l'efficacité. Les résultats des simulations montrent une diminution du coût alimentaire lorsque le niveau de production laitière augmente

Figure 8. Cartes de densité des individus en fonction de leur niveau de production laitière et de leur coût alimentaire à l'échelle de la carrière.

Les individus générés par les simulations S3, S4, S10 et S13 sont systématiquement comparés à ceux de la simulation S2 dont le nuage figure à gauche sur les quatre cartes. Le paramétrage des simulations est récapitulé dans le tableau 2.

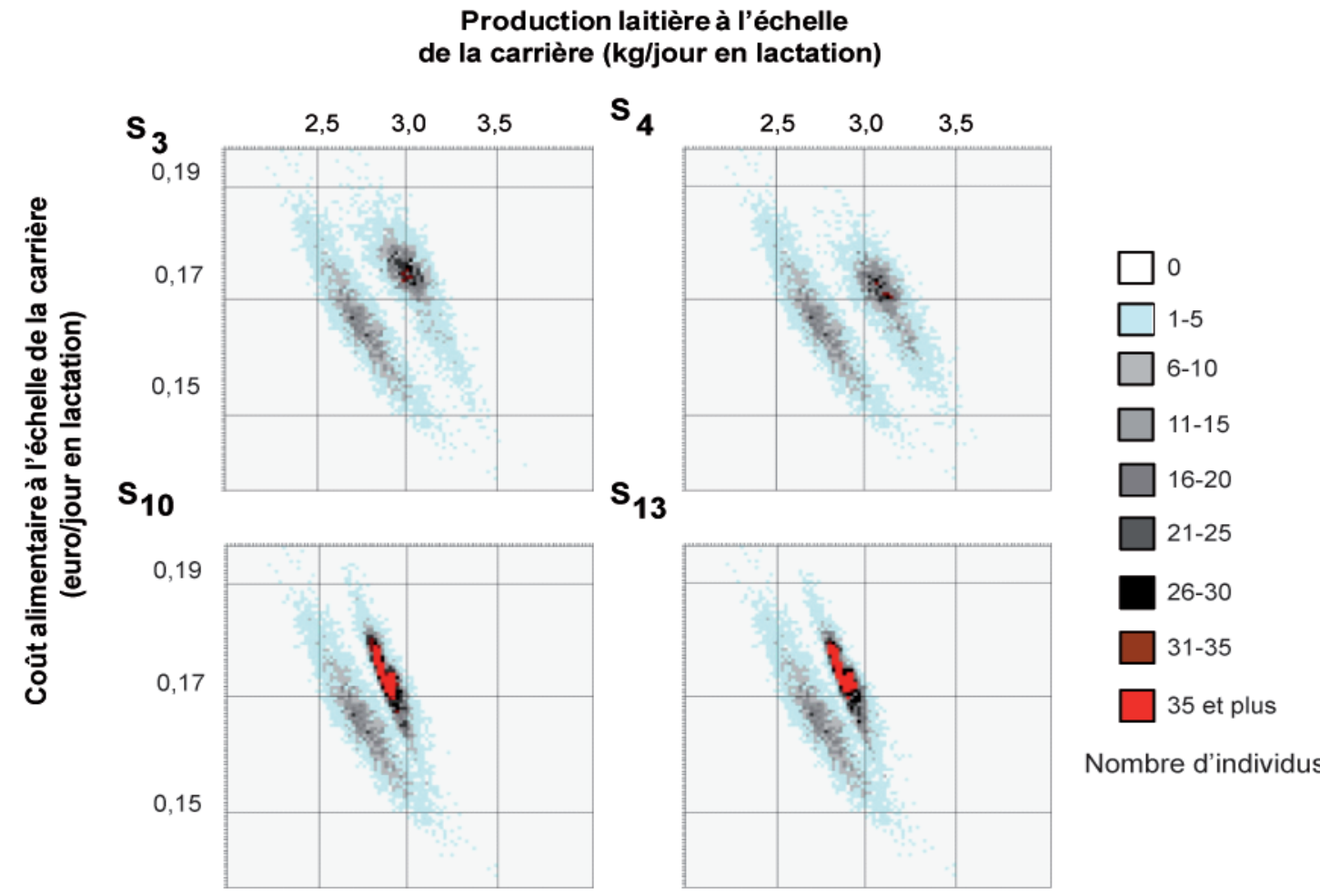


(figure 7). Cette tendance globale traduit à nouveau un effet de dilution des coûts fixes de production par la quantité de lait produit. Cependant, la relation entre coût et production apparaît moins linéaire que la relation entre efficacité et production. Les coûts baissent pour les troupeaux dans une situation de sousalimentation (S2, S11 et S14). Pour atteindre des niveaux de performances équivalents, il est moins coûteux d'utiliser un troupeau avec un potentiel de production laitier élevé (dont les animaux, avec une importante capacité d'ingestion, satisfont leurs besoins avec des aliments encombrants) que d'utiliser une quantité importante d'aliments concentrés avec un troupeau de moins fort potentiel laitier.

\section{3 / Production et efficacité à l'échelle des animaux}

\section{a) Analyse des carrières}

En représentant le fonctionnement de chaque animal au sein du troupeau, le simulateur a permis d'étudier les effets des pratiques sur les performances animales à l'échelle de la carrière. La figure 8 présente le positionnement des individus qui ont généré les performances du troupeau dans l'espace défini par la production laitière et le coût alimentaire à l'échelle de la carrière. L'étude des carrières a porté sur les individus qui effectuaient une carrière complète sur les 10 dernières années de simulation (naissance et sortie du troupeau pendant les 10 dernières années de simulation) et qui effectuaient au moins une lactation. Ainsi, 76569 individus ont été conservés ce qui représentent $65 \%$ environ du total des individus générés. Cette proportion est relativement constante entre les simulations.
Nous avons choisi de présenter les simulations qui conduisaient à des coûts faibles (S2, S3, S4, S10 et S13) pour analyser les individus à l'origine de telles performances. Les individus générés par S2 sont systématiquement comparés aux individus générés par S3, S4, S10 et S13. La forme globale des nuages de points montre à nouveau l'effet de dilution des coûts fixes par le niveau de production laitière. Globalement, plus les individus produisent, plus ils sont efficaces. Au-delà de cette tendance globale, le cœur de la distribution des individus générés par S2 apparaît systématiquement en dessous et plus à gauche que le cœur des distributions des individus générés par S3, S4, S10 et S13. Ainsi, dans S2, la majorité des individus produit moins mais avec un coût faible alors que dans S3, S4, S10 et S13, les individus produisent plus mais avec un coût élevé. Les distributions des simulations $\mathrm{S} 10$ et $\mathrm{S} 13$ présentent des densités d'individus plus fortes que S2 et S4, probablement en lien avec la ration unique testée dans $\mathrm{S} 10$ et $\mathrm{S} 13$. Ces résultats montrent que les effets des pratiques simulées sont cohérents, que l'analyse soit faite au niveau troupeau ou au niveau des carrières individuelles: le positionnement de la majorité des individus détermine les performances au niveau du troupeau. Cependant, la figure 8 illustre l'intérêt du simulateur pour visualiser la variabilité à l'origine des performances. Bien que le grand nombre d'individus qui constitue le cœur de la distribution détermine la performance du troupeau, il existe d'autres individus situés aux bords de la distribution avec des profils de performance différents qui représentent une marge de manœuvre potentielle pour améliorer les performances du troupeau. b) Analyse d'un indicateur biologique individuel

Le simulateur permet d'approfondir l'analyse des effets des pratiques grâce à l'étude de variables individuelles. En effet, certaines variables produites par le simulateur sont des indicateurs du fonctionnement biologique des animaux. Elles permettent de décrire notamment dans quelle mesure les animaux se sont éloignés de leur trajectoire de référence, en lien avec l'apport d'énergie. Ainsi, pour le poids vif, la différence entre la trajectoire réalisée et la trajectoire de référence est déterminée pour chaque animal simulé à 90 jours de sa $2^{\text {ème }}$ lactation (fin de la phase de mobilisation de début de lactation). Ce relevé pondéral en cours de simulation indique si l'animal a accentué la mobilisation de ses réserves, et présente un poids réalisé inférieur au poids de référence (figure 9a) ou s'il a diminué la mobilisation de ses réserves et présente un poids réalisé supérieur au poids de référence (figure $9 b)$. Par exemple, une chèvre simulée qui produit $4 \mathrm{~kg}$ de lait au pic de lactation mobilise $5,8 \mathrm{~kg}$ de réserves corporelles en début de lactation pour exprimer son potentiel. Ce niveau de mobilisation correspond à la trajectoire de référence (pas de contrainte nutritionnelle). En simulant une stratégie de suralimentation, la quantité de réserves mobilisées n'est que de $3 \mathrm{~kg}$. L'alimentation a réduit la mobilisation des réserves de $2,8 \mathrm{~kg}$. En simulant une stratégie de sous-alimentation, la quantité de réserves mobilisées atteint $9,9 \mathrm{~kg}$. L'alimentation a entraîné une augmentation de la mobilisation des réserves de $4,1 \mathrm{~kg}$.

Pour analyser les effets des différentes simulations sur les trajectoires du

Figure 9. Illustration de l'évaluation de l'écart entre le poids vif de référence et le poids vif réalisé pour deux stratégies d'alimentation contrastées simulées avec le modèle de chèvre laitière.

a) Cas d'une mobilisation réduite des réserves corporelles

b) Cas d'une mobilisation accrue des réserves corporelles

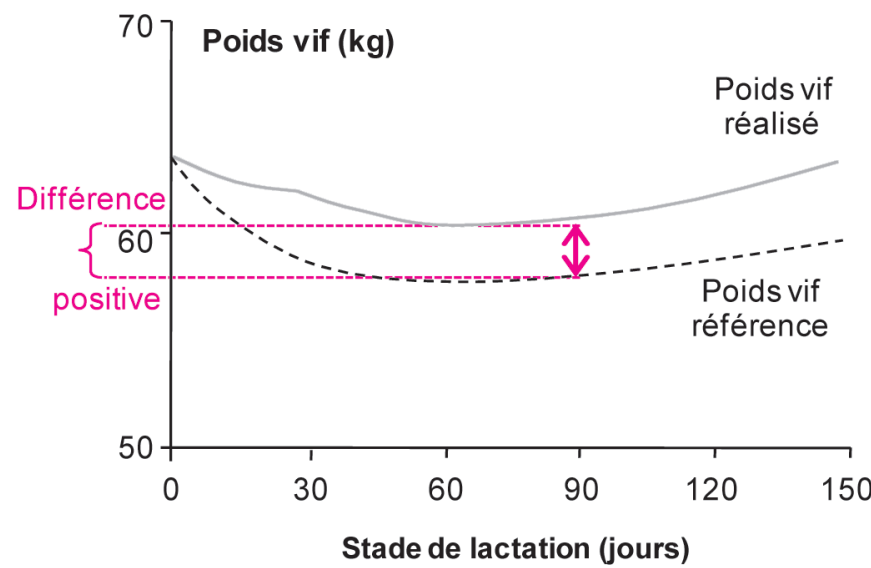

Stade de lactation (jours)

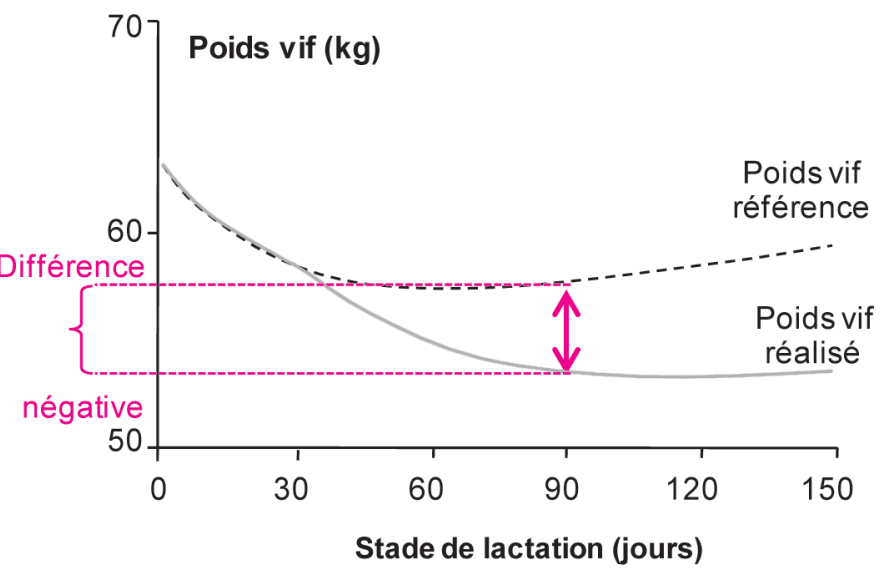

INRA Productions Animales, 2012, numéro 3 
Tableau 3. Répartition des individus du troupeau (\%) selon les cinq types de mobilisation pour les simulations définies au tableau 2. Les types d'individus sont définis par la différence entre leur poids vif de référence (expression du potentiel de production) et

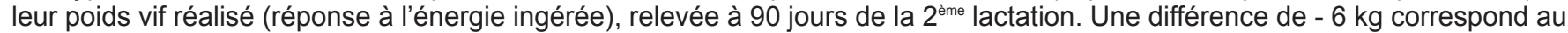
type «mobilisation très forte», une différence comprise entre - 6 et - $2 \mathrm{~kg}$ correspond au type «mobilisation forte», une différence comprise entre $-2 \mathrm{~kg}$ et $+2 \mathrm{~kg}$ correspond au type «équilibre», une différence comprise entre 2 et $6 \mathrm{~kg}$ correspond au type «mobilisation réduite» et une différence supérieure à $6 \mathrm{~kg}$ correspond au type «mobilisation très réduite».

\begin{tabular}{|c|c|c|c|c|c|c|}
\hline Plan & Simulation & $\begin{array}{c}\text { Mobilisation } \\
\text { très forte }\end{array}$ & $\begin{array}{c}\text { Mobilisation } \\
\text { forte }\end{array}$ & Equilibre & $\begin{array}{c}\text { Mobilisation } \\
\text { réduite }\end{array}$ & $\begin{array}{l}\text { Mobilisation } \\
\text { très réduite }\end{array}$ \\
\hline \multirow{4}{*}{1} & S1 & 0,0 & 21,0 & 36,1 & 42,9 & 0,0 \\
\hline & S2 & 0,4 & 88,8 & 9,5 & 1,2 & 0,0 \\
\hline & S3 & 0,0 & 0,3 & 3,4 & 89,9 & 6,3 \\
\hline & S4 & 0,0 & 15,1 & 32,7 & 51,9 & 0,3 \\
\hline \multirow{4}{*}{2} & S5 & 0,0 & 32,6 & 47,2 & 20,2 & 0,0 \\
\hline & S6 & 0,0 & 19,2 & 36,7 & 44,2 & 0,0 \\
\hline & S7 & 0,0 & 36,1 & 44,3 & 19,6 & 0,0 \\
\hline & S8 & 0,0 & 23,8 & 36,1 & 40,1 & 0,0 \\
\hline \multirow{6}{*}{3} & s9 & 0,0 & 54,6 & 39,6 & 5,8 & 0,0 \\
\hline & $\mathrm{S} 10$ & 0,0 & 6,1 & 42,7 & 51,2 & 0,0 \\
\hline & $\mathrm{S} 11$ & 0,3 & 96,9 & 2,8 & 0,1 & 0,0 \\
\hline & $\mathrm{S} 12$ & 0,0 & 54,3 & 40,4 & 5,3 & 0,0 \\
\hline & $\mathrm{S} 13$ & 0,0 & 7,0 & 44,0 & 49,0 & 0,0 \\
\hline & S14 & 0,2 & 97,8 & 2,0 & 0,0 & 0,0 \\
\hline
\end{tabular}

poids vif des individus simulés, cinq cas de figure ont été considérés. Lorsque le poids vif réalisé de l'animal reste dans une fourchette à plus ou moins $2 \mathrm{~kg} \mathrm{du}$ poids vif de référence, l'animal est alors considéré à l'équilibre avec son potentiel de production. Si la différence entre le poids vif réalisé et de référence est inférieure à $-2 \mathrm{~kg}$, l'animal est considéré en mobilisation forte, voire très forte si la différence passe en dessous de - $6 \mathrm{~kg}$. A l'inverse, si la différence poids réalisépoids de référence est supérieure à $2 \mathrm{~kg}$, l'animal est considéré en mobilisation réduite, voire très réduite si la différence dépasse $6 \mathrm{~kg}$.

La notion d'individu à l'équilibre permet d'évaluer les pratiques de conduite simulées sous un nouvel angle. Le tableau 3 présente les proportions d'animaux dans chacun des 5 cas de figure décrits précédemment pour l'ensemble des simulations.

Dans le plan d'expérience $\mathrm{n}^{\circ} 1$, les simulations $\mathrm{S} 1$ et $\mathrm{S} 4$ génèrent des individus variés, répartis dans les types «mobilisation forte», «équilibre» et «mobilisation réduite». A l'inverse, S2 génère des individus majoritairement du type «mobilisation forte» et S3 des individus majoritairement du type «mobilisation réduite», voire «très réduite». Ces profils d'individus sont à relier à la stratégie d'alimentation. Lorsque l'alimentation correspond aux besoins moyens ( $\mathrm{S} 1$ et $\mathrm{S} 4$ ), la répartition des individus simulés entre les types est assez équilibrée. La présence des types «mobilisation forte» et «mobilisation réduite» traduit l'inévitable décalage entre la variabilité intrinsèque des potentiels de production des animaux et le niveau unique d'apport d'énergie par la ration.

Dans le plan d'expérience $\mathrm{n}^{\circ} 2$, les individus sont globalement répartis entre les types «mobilisation forte», «équilibre» et «mobilisation réduite». Cette répartition traduit le fait que dans le plan d'expérience $n^{\circ} 2$, l'alimentation est calée sur les besoins moyens.

Dans le plan d'expérience $\mathrm{n}^{\circ} 3$, les profils d'individus sont contrastés. Dans $\mathrm{S} 9$ et S12, les individus sont majoritairement des types «mobilisation forte» et «équilibre». Dans S10 et S13, les individus sont majoritairement des profils «mobilisation réduite» et «équilibre». Dans S11 et S14, les individus sont majoritairement du type «mobilisation forte». Ces différences entre les simulations sont liées aux stratégies d'alimentation : dans S9 et S12, l'alimentation est calée sur les besoins moyens; dans S10 et S13, 1'alimentation est au-dessus des besoins moyens et dans S11 et S14, l'alimentation est en dessous des besoins moyens.

L'analyse d'un indicateur biologique individuel confirme les effets observés au niveau des carrières et du troupeau. Cette analyse permet également d'approfondir le diagnostic des pratiques.
Les simulations S3, S10 et S13 ont testé le même potentiel de production du troupeau et un niveau d'apports alimentaires au-dessus des besoins moyens. Ces simulations ont conduit au niveau du troupeau à des performances équivalentes (production laitière, efficacité et coût alimentaires). $\mathrm{Au}$ niveau des carrières animales, les distributions des individus de S3, S10 et S13 dans l'espace défini par la production laitière et le coût alimentaire étaient proches. Au niveau des types d'individus simulés, S3, S10 et S13 présentent deux profils de répartition différents. Dans S3, les individus sont majoritairement du type «mobilisation réduite» alors que dans $\mathrm{S} 10$ et S13, ils sont du type «équilibre» et «mobilisation réduite» principalement, voire du type «mobilisation forte». Ce contraste entre d'une part, S3, et d'autre part, $\mathrm{S} 10$ et S13 est à relier au degré de segmentation du plan d'alimentation. Dans les deux cas, les troupeaux sont suralimentés mais pour S10 et S13, la suralimentation a été amortie par la distribution d'une ration unique sur la lactation. Celle-ci a généré un décalage entre l'évolution temporelle des besoins des animaux et l'évolution temporelle des apports. Ainsi, à certains moments, même si la ration est globalement conçue au-dessus des besoins moyens, les animaux ont pu être sous-alimentés. Ce mécanisme explique que S10 et S13 présentent une proportion plus importante d'animaux à l'équilibre que S3. Ainsi, mal- 
gré des performances équivalentes au niveau du troupeau, les mécanismes à l'origine de ces performances étaient différents. Dans S3, une majorité d'animaux sont éloignés de leur trajectoire de référence (expression de leur potentiel de production). Ils sont ainsi plus susceptibles d'évoluer vers des situations de rupture biologique qui présentent un risque potentiel de dégradation des performances. Ainsi, le diagnostic des pratiques a pu être approfondi : la suralimentation des animaux, qui peut être choisie pour sécuriser le système, présente un risque biologique potentiel. Ce risque est diminué si la suralimentation est associée à une ration unique.

\section{3 / Intérêts et limites du simulateur}

L'intérêt du simulateur pour l'évaluation des pratiques de conduite du troupeau caprin laitier repose sur l'intégration de différents niveaux d'organisation (troupeau, lot, animal) et plus particulièrement sur l'intégration du fonctionnement de chaque individu (modèle individu-centré). Ce niveau individuel a permis de représenter explicitement les régulations biologiques à l'origine des réponses animales. Le simulateur permet ainsi d'analyser la variabilité individuelle à l'origine des performances globales du troupeau et d'associer des niveaux de risques biologiques aux pratiques. Cette possibilité renforce l'évaluation des pratiques en permettant de discriminer des conduites qui génèrent des performances équivalentes au niveau du troupeau. Le développement de modèles individu-centré qui représentent explicitement les mécanismes biologiques ouvre des perspectives très intéressantes pour analyser les effets des pratiques de conduite sur les interactions entre fonctions, et plus particulièrement entre les fonctions de nutrition et de reproduction (modèle en vache laitière, (Brun-Lafleur 2011). Le coût de développement (puissance de calcul, volume de données généré) de tels modèles ne doit cependant pas être négligé.

Au-delà de l'intérêt du niveau individuel pour aborder le niveau du troupeau, les approches intégrant plusieurs niveaux d'organisation sont essentielles pour aborder les enjeux auxquels sont aujourd'hui confrontés les systèmes d'élevage. Autour des enjeux sur les rejets environnementaux des élevages (azote, gaz à effet de serre), Faverdin et Peyraud (2010) ont montré que le bénéfice de l'intensification de la production laitière à l'échelle d'une vache n'était pas transposable à l'échelle de l'exploitation agricole ou du pays. Aux échelles agrégées comme le pays, des interactions entre systèmes de production modulent les lois de réponse observées aux niveaux sous-jacents. Plus particulièrement, la co-production de lait et de viande par le troupeau bovin laitier rend plus compliquée l'application de méthodes comme l'analyse de cycle de vie et nécessite d'étendre les frontières du système considéré (Cederberg et Stadig 2003). Autour des enjeux sur la biodiversité, Sabatier (2010) a montré que le compromis entre biodiversité (taille de 2 populations d'oiseaux) et pâturage n'avait pas la même forme à l'échelle de la parcelle et de l'exploitation : la diversité et l'agencement spatial des usages agricoles sont deux leviers d'arbitrage à des échelles supra-parcelle de la relation entre performance écologique et zootechnique. En lien avec l'intérêt des approches à différents niveaux d'organisation, il est également nécessaire aujourd'hui d'appréhender de multiples aspects de la performance des systèmes d'élevage. L'intérêt des réponses multiples aux pratiques a déjà été souligné au niveau de l'animal (Sauvant 1999). Les résultats du simulateur ont montré que l'introduction du coût alimentaire amenait un nouvel éclairage sur les effets des pratiques au niveau du troupeau.

Pour aller plus loin dans la prédiction $\mathrm{du}$ fonctionnement du troupeau caprin, un certain nombre de développements seront nécessaires.

Le modèle de gestion technique a été conçu pour pouvoir représenter différentes formes d'organisation du troupeau en lots, en lien avec différents projets de production. Pour l'instant, la version informatique du simulateur intègre uniquement l'organisation d'une période de mise bas au printemps (livraison de lait centrée sur le printemps). L'implémentation informatique des projets de livraison de lait centrée sur l'automne et de livraison de lait régulière permettra de tester si les effets des leviers niveau d'alimentation et potentiel de production du troupeau sont les mêmes.

Le modèle de chèvre laitière simule les réponses à l'alimentation sur la base d'un système d'allocation de l'énergie ingérée aux différentes fonctions physiologiques. Une des limites du modèle est de ne pas considérer les apports protéiques. Ces derniers peuvent moduler les réponses à l'énergie ingérée en cas de taux azoté insuffisant, ce qui est assez rare dans nos systèmes de production. Ils représentent une sortie d'intérêt, à la fois par rapport à l'efficacité du troupeau et également par rapport aux rejets dans l'environnement (perte d'azote). Il serait également intéressant d'approfondir la représentation des priorités entre fonctions, notamment sur la base des modèles développés en vache laitière (Martin et Sauvant 2010a, 2010b). Pour l'instant, le modèle de chèvre laitière n'intègre pas d'interactions entre les fonctions de nutrition et de reproduction. Le niveau des réserves n'a pas d'effet sur la probabilité de fécondation. Les résultats des simulations au niveau des carrières individuelles sous-estiment donc probablement les effets des stratégies d'alimentation. En effet dans le simulateur, le niveau des réserves a un effet uniquement sur la production laitière, alors que l'aptitude à la reproduction pourrait aussi être affectée et plus globalement la longévité dans le troupeau. Des travaux en bovins allaitants ont montré que le taux de survie dans le troupeau est affecté par la stratégie d'alimentation (Blanc et al 2004).

Les priorités entre fonctions physiologiques devront également intégrer la variabilité individuelle pour simuler différentes règles de partition de l'énergie ingérée. Dans l'état actuel, la règle de pénalisation de la production laitière en fonction du niveau de sollicitation des réserves et du déficit énergétique est la même pour tous les individus simulés. Cependant, cette capacité à s'adapter à un déficit est variable : certains animaux peuvent avoir tendance à maintenir une production élevée en sollicitant leurs réserves alors que d'autres maintiennent leur niveau de réserves en baissant leur niveau de production.

Ces pistes de développement du modèle animal font apparaître un besoin d'expérimentations spécifiques visant à quantifier les capacités d'adaptation des chèvres. Ces expérimentations devront permettre de quantifier la variabilité individuelle des réponses ainsi que les effets à long terme de certaines adaptations biologiques (effet rémanent du niveau des réserves corporelles, par exemple). Des travaux amorcés dans différentes équipes INRA vont dans ce sens (UE INRA Rouillé : projet Patuchev; UMR MoSAR : Schmidely et al 2011). Les données expérimentales autour des capacités d'adaptation individuelles permettront d'appréhender les seuils de rupture biologique et donc de quantifier la notion de risque biologique associée à certaines conduites. La simulation du fonctionnement du troupeau pourra alors tester les réponses du système à des perturbations externes (aléa sur les ressources alimentaires, par exemple) et caractériser ainsi les pratiques de conduite à même d'accroître la robustesse du système d'élevage.

\section{Conclusion}

Simuler le fonctionnement du troupeau caprin laitier a permis de quantifier les effets du potentiel de production du troupeau, des pratiques d'alimentation et de reproduction sur la production lai- 
tière et l'efficacité alimentaire du troupeau. Le niveau des apports alimentaires et le potentiel de production ont des effets importants sur la production et l'efficacité via un phénomène de dilution des coûts nutritionnels fixes de production. Les résultats ont également montré que le découpage du plan d'ali- mentation modulait les effets du niveau des apports alimentaires. L'analyse d'indicateurs fins du fonctionnement biologique des individus du troupeau a permis d'approfondir les mécanismes à l'origine des performances, ce qui a permis de discriminer des conduites qui a priori généraient des performances équivalentes au niveau du troupeau. Ces conduites sont en fait associées à des niveaux de risques biologiques différents. Lors de situations de perturbations de l'environnement de l'élevage, ces risques sont susceptibles de donner lieu à des capacités d'adaptation du troupeau différentes.

\section{Références}

Blanc F., Bocquier F., Debus N., Agabriel J., D'Hour P., Chilliard Y., 2004. La pérennité et la durabilité des élevages de ruminants dépendent des capacités adaptatives des femelles. Inra Prod. Anim., 17, 287-302.

Brun-Lafleur L., 2011. Modélisation du fonctionnement d'un troupeau laitier pour anticiper les conséquences de son pilotage. Thèse de doctorat, AgroCampus Ouest, Rennes, France, 280p.

Cederberg C., Stadig M., 2003. System expansion and allocation in life cycle assessment of milk and beef production. Int. J. Life Cycle Assessment, 8, 350-356.

Coulon J.B., Lescourret F., Faye B., Landais E., Troccon J.L., Perochon L., 1993. Description de la base de données «LASCAR», un outil pour l'étude des carrières des vaches laitières. Inra Prod. Anim., 6, 151-160.

Coulon J.B., Perochon L., Lescourret F., 1995. Pattern of milk production and live weight of dairy cows during their successive lactations. Ann. Zoot., 44, 189-199.

Cournut S., Dedieu B., 2004. A discrete events simulation of flock dynamics: a management application to three lambings in two years. Anim. Res., 53, 383-403.

Faverdin P., Peyraud J.L., 2010. Nouvelles conduites d'élevage et conséquences sur le territoire : cas des bovins laitiers. In : Publications de l'Académie d'Agriculture de France. Elevages intensifs et environnement. Les effluents : menace ou richesse? Editions Académie d'Agriculture de France, Paris, France, 89-100.

Friggens N., Newbold J.R., 2007. Towards a biological basis for predicting nutrient partitioning: the dairy cow as an example. Animal, 1,8797.

Institut de l'Elevage, 2008. Hausse du prix des aliments, des pistes pour alléger les charges. http://www. inst-elevage. asso.fr/html1/spip.php? article16615.

Gibon A., 1994. Landscape preservation objectives and the sheep flock management in Meditarranean moutains. Proceedings of a meeting held within the technical consultation of the FAO-CIHEAM Network on Cooperative Sheep and Goat Research, Thessaloniki, Grèce, 188-193.

Guérin G., Bellon S., 1989. Analysis of the functions of pastoral areas in forage systems in the Mediterranean region. Etudes Rech. Syst. Agraires Dév., 16, 147-156.

Landais E., 1987. Recherches sur les systèmes d'élevage. Questions et perspectives. Document de travail INRA SAD, Versailles, France, $75 \mathrm{p}$.

Lasseur J., Landais E., 1992. Mieux valoriser l'information contenue dans les carnets d'agnelages pour évaluer des performances et des carrières de reproduction en élevage ovin- viande. Inra Prod. Anim., 5, 43-58.

Lee G.J., Atkins K.D., Sladek M.A., 2009. Heterogeneity of lifetime reproductive performance, its components and associations with wool production and liveweight of Merino ewes. Anim. Prod. Sci., 49, 624-629.

Martin O., Sauvant D., 2010a. A teleonomic model describing performance (body, milk and intake) during growth and over repeated reproductive cycles throughout the lifespan of dairy cattle. 1. Trajectories of life function priorities and genetic scaling. Animal, 4, 2030-2047.

Martin O., Sauvant D., 2010b. A teleonomic model describing performance (body, milk and intake) during growth and over repeated reproductive cycles throughout the lifespan of dairy cattle. 2.Voluntary intake and energy partitioning. Animal, 4, 2048-2056.

Peyraud J.L., Le Gall A., Delaby L., Faverdin P., Brunschwig P., Caillaud D., 2009. Quels sys- tèmes fourragers et quels types de vaches laitières demain? Fourrages, 197, 47-70.

Puillet L., 2010. Modéliser la variabilité biologique en réponse aux pratiques de conduite. Application au troupeau caprin laitier. Thèse de doctorat, AgroParisTech, 252p.

Puillet L., Martin O., Tichit M., Sauvant D., 2008. Simple representation of physiological regulations in a model of lactating female: application to the dairy goat. Animal, 2, 235-246.

Puillet L., Martin O., Sauvant D., Tichit M., 2010. An individual-based model to simulate individual variability and herd long term performance. Animal, 4, 2084-2098.

Puillet L., Martin O., Sauvant D., Tichit M., 2011. Introducing efficiency into the analysis of individual lifetime performance variability: a key to assess herd management. Animal, 5, 133153.

Sabatier R., 2010. Arbitrages multi-échelles entre production agricole et biodiversité dans un agroécosystème prairial. Thèse de doctorat, AgroParisTech, Paris, France. 228p.

Sauvant D., 1999. Le concept de lois de réponses multiples aux régimes, trait d'union entre les domaines techniques et économiques de l'élevage. Renc. Rech. Rum., 6, 11-17.

Sauvant D., Giger-Reverdin S., Meshy F., 2007. Alimentation des caprins. In : Alimentation des bovins, ovins et caprins. Editions QUAE, Versailles, France, 137-148.

Schmidely P., Duvaux-Ponter C., LaporteBroux B., Tessier J., Friggens N., 2011. Characterizing individual differences in performance responses to a nutritional challenge. In Book of abstracts of the $62^{\text {nd }}$ EAAP Meeting, Stavanger, Norvège, $\mathrm{p} 82$.

Vandehaar M.J., 1998. Efficiency of nutrient use and relationship to profitability on dairy farms. J. Dairy Sci., 81, 272-282. 


\title{
Résumé
}

Cet article présente un simulateur du fonctionnement du troupeau caprin laitier qui permet d'évaluer les effets des pratiques de conduite sur les performances du troupeau. Le simulateur est basé sur l'interaction entre un système biotechnique et un système décisionnel. Le système biotechnique représente chaque animal grâce à un modèle de chèvre laitière. Le système décisionnel représente les opérations techniques d'alimentation, de reproduction et de renouvellement. Trois plans d'expériences ont testé les effets $i$ ) du niveau des apports alimentaires et du potentiel de production du troupeau, ii) de la segmentation du plan d'alimentation et de la durée de la lutte et iii) d'une conduite simplifiée du troupeau. Le simulateur a permis d'évaluer la production laitière et l'efficacité alimentaire à l'échelle du troupeau et des individus. Le niveau des apports et le potentiel de production du troupeau ont des effets marqués sur la production et l'efficacité alimentaire via la dilution des coûts de production par la quantité de lait produite. La segmentation du plan d'alimentation module les effets du niveau des apports alimentaires. L'examen d'indicateurs biologiques individuels permet de distinguer des conduites a priori équivalentes en termes de performance du troupeau. Ces conduites sont en fait associées à des niveaux de risques biologiques différents, susceptibles de moduler les capacités d'adaptation du troupeau en réponse à une perturbation.

\begin{abstract}
A model of the herd functioning to assess the milk production and the feed efficiency in dairy goat systems

This paper describes a dairy goat herd model, which simulates the effects of management practices on herd performance. The model is based on the interaction between a decisional system and a biological system. The biological system represents each animal based on a dairy goat model. The decisional system is based on the technical operations of feeding, reproduction and replacement. Three experimental plans were run to test the effects of i) the energy level of the diet and the mean herd production potential; ii) the number of steps within the feeding plan and the length of the breeding season and iii) a simplified management of the herd. The model enables assessing milk production and feed efficiency at both herd and individual levels. The results showed that the feeding level and the mean herd potential have marked effects on milk production and feed efficiency through a dilution effect of the production cost by the quantity of milk produced. The results also showed that the degree of segmentation of the feeding plan modulates the effects of the feeding level. By looking at biological individual variables, it was possible to discriminate management practices which led to similar performances at the herd level. These practices generated different biological risks, which can potentially modulate herd adaptive abilities to environmental perturbations.
\end{abstract}

PUILLET L., TICHIT M., MARTIN O., SAUVANT D., 2012. Apport d'un modèle du fonctionnement du troupeau pour évaluer la production laitière et l'efficacité alimentaire en élevage caprin. In : Elevage caprin. Baumont R., Sauvant D. (Eds). Dossier, INRA Prod. Anim., 25, 291-304. 